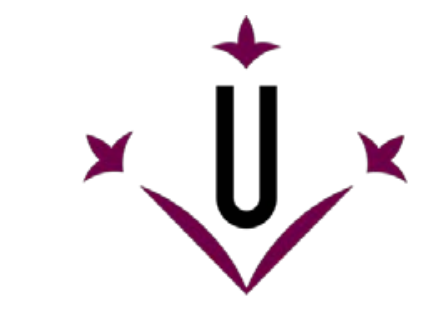

Universitat de Lleida

Document downloaded from:

http://hdl.handle.net/10459.1/65075

The final publication is available at:

https://doi.org/10.1007/s10681-006-9310-5

Copyright

(c) Springer Verlag, 2007 


\section{Genetic markers for doubled haploid response in barley}

Xi-Wen Chen ${ }^{1,3}$, Luís Cistué ${ }^{1}$, María Muñoz-Amatriaín ${ }^{1}$, Miguel Sanz ${ }^{1}$, Ignacio Romagosa ${ }^{2}$, Ana-María Castillo ${ }^{1}$ and María-Pilar Vallés ${ }^{1 \rrbracket}$

(1) Departamento de Genética y Producción Vegetal, Estación Experimental Aula Dei, Consejo Superior de Investigaciones Científicas, Apdo 202, 50059 Zaragoza, Spain

(2) Area de Conreus Extensius, Centre UdL-IRTA, 25198 Lleida, Spain

(3) Present address: Department of Biochemistry and Molecular Biology, Nankai University, Tianjin, 300071, People's Republic of China

M.P. Vallés

Email: valles@eead.csic.es 
Abstract In order to analyse the genetic control of anther culture response in barley, a doubled haploid $(\mathrm{DH})$ population from the cross between a medium responsive cultivar 'Dobla' and the model cultivar 'Igri' was produced. A linkage map was constructed with 91 markers. A sub-population of 41 lines was characterised for different components of the anther culture response, and was used for quantitative trait loci (QTL) analysis. The vrs1 locus region on chromosome $2 \mathrm{H}$, which determines inflorescence row type, was coincident with the largest putative QTL for number of embryos (nEMB) and albino plants. A region of chromosome $6 \mathrm{H}$ was associated with QTLs for nEMB and green plants. QTLs for number and percentage of green plants were located on the long arm of chromosome 5H. Therefore, new QTLs for main components of barley anther culture response were identified on chromosomes $2 \mathrm{H}, 5 \mathrm{H}$ and $6 \mathrm{H}$, indicating that anther culture response in barley could be controlled by relative few genes of large effect. This work is a useful step towards the identification of new regions on the barley genome that could be associated with fundamental biological process implicated in the anther culture response.

Keywords Anther culture, barley, doubled haploid (DH), microspore embryogenesis, QTLs 


\section{INTRODUCTION}

Doubled haploidy (DH) has many applications in plant research and is particularly attractive to plant breeders because it provides a rapid way to produce large numbers of homozygous plants at any stage in a breeding programme, significantly reducing the time to release new cultivars.

Microspore embryogenesis via anther and isolated microspore cultures has been widely used and well-developed in barley, therefore new barley varieties are continuously being released from DH lines (Devaux and Pickering 2005). DHs have also been fundamental to the development of genetic maps and quantitative trait loci (QTL) analysis (Forster and Thomas 2003). The combination of DH techniques with other molecular tools such as marker assisted selection (Forster and Thomas 2005) mutagenesis (Szarejko 2003; Castillo et al. 2001b), or transformation have been also reported (Horvath et al. 2002).

Barley exhibits genotypic dependency to $\mathrm{DH}$ procedures resulting in a limitation to the breeders as several cultivars presented a low response to this technique (Castillo et al. 2001c). This dependency promote a continuous interest for optimizations of protocols (Jacquard et al. 2006), to identify genes implicated in the process and to map loci controlling the microspore embryogenesis response (Devaux and Pickering 2005).

Early studies on the genetic control of the microspore embryogenesis revealed certain complexity of the trait (Foroughi-Wehr et al. 1982; Powell 1988; Larsen et al. 1991; Hou et al. 1994), as several nuclear genes, with additive and dominance effects, acted independently in each of the steps of the procedure and frequently interacted with environmental conditions. Specifically, the ability to produce embryos and green plant regeneration were under strong genetic controls, but, no genetic correlation was observed between them. Additivity was the primary contributor to the observed response for embryoid formation and total plant regeneration, while dominance accounted for a slightly larger proportion of the green plant regeneration response.

Lately differential gene expression studies were carried out to recognize candidate genes specifically expressed during the process, but only a few genes have been identified in cereals: a 32-kDa sporophytic protein in maize (Vergne et al. 1993), an ABA-responsive cysteine-labelled metallothionein gene in wheat (Reynolds and Crawford 1996), and a glutathione S-transferase, a non-specific lipid transfer protein and an unknown protein belonging to the arabinogalactan- like protein (AGP) in barley 
(Vrinten et al. 1999) and endosperm-specific proteins ZmAE and ZmAE3 in maize (Magnard et al. 2000). Recently genomic approaches have been undertaken to study gene expression associated to early steps of the process (Maraschin et al. 2006; MuñozAmatriaín et al. 2006) identifying genes related mainly with changes in metabolism and stress response.

The use of genetic markers provides a powerful approach in the identification and location of relevant loci responsible for genetic variability of quantitatively inherited traits. A great deal of effort has been devoted to identify molecular markers linked to genetic factors associated with the anther culture response in cereals such as maize (Cowen et al. 1992; Murigneux et al. 1994; Beaumont et al. 1995), rice (He et al. 1998; Yamagishi et al. 1998; Kwon et al. 2002) and wheat (Torp et al. 2001, 2004). However, Manninen (2000) reported a unique study in barley where QTLs for percentage of responsive anthers, plants per responsive anther and spontaneous diploidization were identified on barley chromosomes $2 \mathrm{H}, 3 \mathrm{H}$ and $4 \mathrm{H}$.

The objective of this study was to contribute to a better understanding of the genetic control of anther culture response in barley, and the determination of putative QTLs associated with the ability to produce embryos and the percentage of green plant regeneration, the most critical components of the DH process, that still remained unmapped.

\section{MATERIALS AND METHODS}

\section{Plant material and anther culture}

The winter/spring six-rowed Dobla and the winter two-rowed Igri cultivars were used for this study. Igri is a model variety for microspore embryogenesis studies due to its good response under both microspore and anther culture. Dobla is an agronomically important cultivar in Spain with an intermediate response to anther culture (Cistué et al. 1999). A population of 67 DH lines was obtained from a cross between Dobla and Igri, by anther culture, and used for linkage map construction. A random sub-population of 41 lines was used for QTL mapping, due to the difficulties for a large population anther culture response characterization. 
A well-established standard protocol described by Cistué et al. (2003) was followed for evaluation of the anther culture response. Dissected anthers were pretreated with $0.7 \mathrm{M}$ mannitol for 4 days at $25^{\circ} \mathrm{C}$ in the dark. After the pre-treatment, ten anthers from each side of the spike were cultured in $1.5 \mathrm{ml}$ liquid FHG medium supplemented with $200 \mathrm{~g} \mathrm{l}^{-1}$ of Ficoll Type-400 (Sigma, Deisenhofen, Germany). The plates were replenished with another $1.5 \mathrm{ml}$ of FHG medium supplemented with $400 \mathrm{~g} \mathrm{l}^{-1}$ Ficoll, after $12-15$ days of culture. Cultures were kept at $25^{\circ} \mathrm{C}$ in the dark. Thereafter, well-developed embryos (21-40 days of culture) were transferred to FHG medium without glutamine and supplemented with $31 \mathrm{~g} \mathrm{l}^{-1}$ maltose, $2.9 \mu \mathrm{M}$ indole acetic acid (IAA), $4.4 \mu \mathrm{M}$ benzylaminopurine (BA), and $3 \mathrm{~g}^{-1}$ Phytagel, for plant regeneration.

Variables related to DH production included the number of dividing microspores (nDM) (recorder under a stereoscopic microspcope by counting, in one tenth of the Petri dish area, microspores which underwent division and further developed into globular embryos), the number of embryos (nEMB), green plants (nGP) and albino plants (nAP) (all referred to 100 cultured anthers), were considered. The variable pGP (number of green plants per total plants), was also recorded as a measure of the albino ratio.

\section{Molecular markers analysis}

For RAPD analysis, amplified products were resolved by electrophoresis in 1.52\% agarose gels, and recorded with a BioRad Gel Doc 2000 system. Of the random decamer primers included on kits E, F, J, X, AD, AN, AS (Operon Technologies, Cologne, Germany) used for polymorphism selection, only those primers with strong and repetitive polymorphic band(s) were used for mapping.

STS primers were purchased from Amersham Pharmacia Biotech, Freiburg, (Germany), according to the published sequences (Blake et al. 1996; Sayed-Tabatabaei et al. 1999). Amplification reactions were optimised following the primer-specific recommendations. Polymorphism was checked without enzyme digestion and with four different restriction enzymes: HaeIII, TaqI, HhaI and MspI. Other restriction enzymes, HinfI, AccII, ScrFI, NdeII and AvaII were also used if specified on the references. Digested fragments were resolved in 3\% agarose gels.

SSR primers were purchased from Invitrogen Life Technologies, Paisley, (UK), according to the sequences appeared in Becker and Heun (1995), Liu et al. (1996) and 
Ramsay et al. (2000). The amplification reaction was carried out according to the specific recommendations from the above references. Amplification products were resolved in 3\% agarose gels, or in $6 \%$ polyacrylamide gels (7 M Urea) with silver staining (Promega Corporation, Mannheim, Germany) and recorded with a Bio-Rad FX-phosphorimager. Some SSR loci showed the presence of the Igri allele and a size variation of the Dobla parental allele, in the DH population. In all cases, and after linkage analysis with the nearest marker, the 'no parental' alleles were treated as alleles of Dobla.

\section{Statistical analysis}

Linkage analysis of the molecular and morphological traits data was performed using the software package MAPMAKER Version 3.0 (Lander et al. 1987). SSRs with known chromosomal locations, based on previously published maps were used as anchor markers to assign linkage groups to chromosomes. Chi-square was used to test for alleles deviations from the expected 1:1 segregation ratio. For QTL analyses, markers with severe segregation distortion were excluded from the map. Linkage groups and map distances were then recalculated.

Differences in anther culture variables between the two parents have been tested with ten replications of ten anthers within the same batch of plants and simultaneously with the DH population.

Significance thresholds for LOD-scores of QTL were estimated from 3,000 permutations for simple interval mapping (SIM) and simplified composite interval mapping (sCIM) procedures of the software package MQTL (Tinker and Mather 1995). Significant QTLs were considered when there were coincident peaks with both SIM and sCIM analysis, and the SIM peaks exceeded the significance thresholds $(P<0.05)$. Estimates of the positions of QTL corresponded to the peaks of the sCIM scans. Individual and joint additive effects of QTL were used to estimate the percentage of

phenotypic variation $\left(R^{2}\right.$ and $\left.\mathrm{mR}^{2} \mathrm{p}\right)$ for the significant QTLs, and confirmed by stepwise multiple regression analysis. Other statistical test procedures were performed using the SAS/STAT statistics software package (SAS Institute Inc. Cary, NC, USA).

\section{RESULTS}




\section{Variation in anther culture traits}

The parental cultivars Dobla and Igri differed significantly in all components of the anther culture response except in the number of $\mathrm{nDM}$ (Table $\underline{1}$ ). Igri showed the highest values for all traits apart from the nAP. These results are similar to previous characterizations of parental cultivars, but only data collected at the same time and conditions of the DH population were presented. The DH population had medium values close to Dobla, the parental with the lower anther culture response. There were negative and positive transgressive segregants for most traits studied in the population.

\section{Linkage map construction}

In order to determine the polymorphic markers between cultivars Dobla and Igri, 104 RAPD primers were checked and 23 of them (22.1\%) produced 45 polymorphic bands. Among 48 STS markers, 17 (35.4\%) showed polymorphism at least with one restriction enzyme, one marker showed presence or absence difference and one marker showed a size difference. SSR markers presented the highest degree of polymorphism, with $39.2 \%$ (51 out 130 markers). After discarding not assigned or confusing linkage markers, the map had a total length of $1,228 \mathrm{cM}$ and included 91 markers: 35 RAPD markers, 13 STS markers, 42 SSR and the row-type morphological marker vrs1 (Fig. 1). Markers were grouped in 11 linkage groups assigned to the seven barley chromosomes. RAPD markers tended to cluster in some regions and particularly in some chromosomes.

Unmapped regions were located on the long arm of chromosomes $1 \mathrm{H}, 3 \mathrm{H}, 5 \mathrm{H}$, $6 \mathrm{H}$ and $7 \mathrm{H}$, and on the centromeric region of $7 \mathrm{H}$. Markers with a significant distortion of segregation (about 15\%), were distributed in all chromosomes except on 3H (Fig. 1), and in most cases an excess of alleles of the more favourable genotype, Igri, was observed. After the elimination of these markers for QTL analysis, the map comprised 68 markers covering $996.9 \mathrm{cM}$.

\section{QTLs for anther culture response}


One or two significant QTLs were identified for each component of the anther culture response in barley, apart from the number of nDM (Table 2). On the long arm of chromosome $2 \mathrm{H}$, a QTL with large effect on the nEMB and a minor one for nAP were located close to the centromere (Fig. 1), explaining 40.92 and $24.94 \%$ of the phenotype variance, respectively. The nearest marker locus was located, in both QTLs, on/or close to the vrs1 locus, which determines inflorescence row type (Table 2). Markers that presented the strongest distortion of segregation found in this study were located at the edges of these $2 \mathrm{H}$ QTLs intervals, with selection for the Igri allele.

Two QTLs with short intervals were located on the centromeric region of chromosome $6 \mathrm{H}$ (Fig. 1). These QTLs were associated with the nEMB and nGP, with unequal effect on the percentage of phenotype variance, 11.54 and $32.68 \%$, respectively (Table 2). The nearest marker locus in both QTLs was the microsatellite EBmac602. In this case, there was no significant selection for any of the parental alleles on the markers at these QTls intervals.

Significant QTLs for the nGP and pGP were located on the long arm of chromosome $5 \mathrm{H}$ on an area with few polymorphic markers, therefore difficult to map (Fig. 1). The nearest marker locus interval was the same in both QTLs, and a similar effect on the percentage of phenotype variance was observed for the nGP (17.85\%), and for the pGP (21.07\%) (Table 2). No markers with distortion of segregation were identified in this marker interval.

Estimated joint additive effects of QTLs for nEMB and nGP were significant (Table 2). The lowest percentage of phenotypic variance was explained for pGP (21.07\%), whereas the highest was for nEMB (52.47\%) and nGP (50.53\%). Presence of the Igri allele increased the values of all variables except the nAP.

\section{DISCUSSION}

In this study we have identified three chromosomal regions implicated in the barley anther culture process, located on chromosomes $2 \mathrm{H}, 5 \mathrm{H}$ and $6 \mathrm{H}$. In each chromosomal region two significant QTLs for components of anther culture response, except for the number of $\mathrm{nDM}$, were determined.

Quantitative trait loci with large effect were located on chromosome $2 \mathrm{H}$ on/or close to the vrs1 locus, and explained 40.92 and $24.94 \%$ of the phenotype variance of 
$\mathrm{nEMB}$ and nAP, respectively. Interestingly associations among the percentage of responsive anthers and number of total plants regenerated, and molecular markers on the centromeric region of barley chromosome $2 \mathrm{H}$ have been described in barley before (Manninen 2000). Also, a homologous region on chromosomes of wheat could play a mayor role in the control of pGP in anther culture (Torp et al. 2001). Associations of this chromosomal region with different phenomena implicated in anther culture in different systems could arise from the control of some general mechanism for the morphogenic functions. In addition, QTLs for callus growth, shoot differentiation (Shd1 and Qsr1) (Komatsuda et al. 1993; Mano et al. 1996), and green plant regeneration (Bregitzer and Campbell 2001) from immature embryos callus were also mapped close to vrs1 locus, and different agronomic characters related to reproductive capacity have been associated with it.

In our study, markers close to the QTLs on chromosome $2 \mathrm{H}$ presented the strongest distortion of segregation with selection for the Igri allele. But this selection was not always correlated with a high-anther culture response, indicating strong recombination rates in this area. This situation was coincident with the physical map of Kuenzel et al. (2000), where the vrs1 locus was located in one region with the highest recombination rates of the $2 \mathrm{H}$ chromosome. It has been demonstrated, in a comparative study of an Igri six-rowed induced mutant versus the two-rowed isogenic Igri, that there were no significant differences in anther culture response suggesting also a linkage effect of the vrs1 locus with the candidate genes for this QTL (Castillo et al. 2001a).

The centromeric region of chromosome $6 \mathrm{H}$ was associated with the $\mathrm{nEMB}$ and the nGP, with unequal effect on the percentage of phenotype variance, 11.54 and $32.68 \%$, respectively. The presence of the Igri allele increases the values of all these traits. None QTL associated with anther culture response in cereals has been previously described on this region. Only a QTL located on the short arm of chromosome 6R was associated with regeneration rate in anther culture of rye (Grosse et al. 1996). The QTL located on chromosome $6 \mathrm{H}$ appears analogous to the one identified for green plant regeneration from somatic tissue culture of barley (Qsr3, Mano et al. 1996; Bregitzer and Campbell 2001).

In this study, a significant QTL on chromosome $5 \mathrm{H}$ was identified for $\mathrm{pGP}$ and the nGP. The presence of a QTL for pGP has been reported previously on chromosome 5BL of wheat (Torp et al. 2001), 5RL of rye (Grosse et al. 1996), and homoeologous chromosome 9 of rice (He et al. 1998). QTLs for green plant regeneration from barley 
callus were mapped close to our QTL position (Qsr4, Mano et al. 1996; Bregitzer and Campbell 2001).

The results presented in this study revealed associations between main components of anther culture response and QTLs. Up to $50.53 \%$ of phenotypic variance could be explained for the variable nGP by the additive QTLs effects on chromosomes $6 \mathrm{H}$ and $5 \mathrm{H}$. This variable reflects the final efficiency of the doubled haploid production process. Substantial portion of the genotypic variance remained unexplained for pGP, considering that only the QTL on chromosome $5 \mathrm{H}$ was statistically significant $(21.07 \%$ phenotypic variance), whereas Larsen et al. (1991) described that up to a $76 \%$ of total variation depended on the genotype, suggesting that more QTLs segregate in this cross, but the conditions of the study mask the associations.

All these results indicate that only three major chromosomal regions are involved in the control of the barley anther culture process, for the different variables studied. Common and different genetic controls were implicated along the process. Some QTLs were at similar positions to those detected in other barley population and/or in other tissue culture process, or in anther culture response of related cereals. Although this study was limited by the number of genotypes that could be analysed, running the risk of over/underestimating genetics effects of QTLs, this work is a useful step towards the identification of new regions of the genome associated with anther culture in barley. Further work should be done to clarify the 2H QTL effects, and identify new chromosomal regions associated with the pGP, which still greatly limits the potential of anther culture in barley.

Acknowledgements X-W. Chen was recipient of a fellowship form the Ministerio de Educación y Cultura from the Spanish government. The research was supported by Projects PB97-1159, and AGL2001-1631 from Plan Comisión Interministerial de Ciencia y Tecnología of Spain.

\section{REFERENCES}

Beaumont VH, Rocheford TR, Widholm JM (1995). Mapping the anther culture response genes in maize (Zea mays L.). Genome 38: 968-975. 
Becker J, Heun M (1995). Barley microsatellites: allele variation and mapping. Plant Mol Biol 27: 835-845.

Blake TK, Kadyrzhanova D, Shepherd KW, Islam AKMR, Langridge PL, McDonald CL, Erpelding J, Larson S, Blake NK, Talbert LE (1996). STS-PCR markers appropriate for wheat-barley introgression. Theor Appl Genet 93: 826-832.

Bregitzer P, Campbell RD (2001). Genetic markers associated with green and albino plant regeneration from embryogenic barley callus. Crop Sci 41: 173-179.

Castillo AM, Cistué L, Romagosa I, Vallés MP (2001a). Low responsiveness of sixrowed genotypes to androgenesis in barley does not have a pleiotropic basis. Genome 44: 936-940.

Castillo AM, Cistué L, Vallés MP, Sanz JM, Romagosa I, Molina-Cano JL (2001b). Efficient production of androgenic doubled haploid mutants in barley by the application of sodium azide to anther and microspore cultures. Plant Cell Rep 20: $105-111$.

Castillo AM, Vallés MP, Cistué L (2001c). Improvements of barley androgenesis for plant breeding. In: Bohanec B (ed.) Biotechnological approaches for utilization of gametic cells. Office for Official Publications of the European Communities, Luxembourg, pp 15-21.

Cistué L, Ramos A, Castillo AM (1999). Influence of anther pretreatment and culture medium composition on the production of barley doubled haploids from model and low responding cultivars. Plant Cell Tiss Org Cult 55: 159-166.

Cistué L, Vallés MP, Echávarri B, Sanz JM, Castillo AM (2003). Barley anther culture. In: Malupszynski M, Kasha K, Foster B (eds) Doubled haploid production in crop plants. A Manual. FAO/IAEA Division, Wien, pp 29-35.

Cowen NM, Johnson CD, Armstrong K (1992). Mapping genes conditioning in vitro androgenesis in maize using RFLP analysis. Theor Appl Genet 84: 720-724.

Devaux P, Pickering R (2005). Haploids in the improvement of Poaceae. In: Palmer CE, Keller WA, Kasha KJ (eds) Haploids in crop improvement II. Biothechnology in Agriculture and Forestry 56. Springer, Berlin Heidelberg New York, pp 215242.

Foroughi-Wehr B, Friedt W, Wenzel G (1982). On the genetic improvement of androgenic haploid formation in Hordeum vulgare L. Theor Appl Genet 62: 233-239. 
Forster BP, Thomas WTB (2003). Doubled haploids in genetic mapping and genomics. In: Malupszynski M, Kasha K, Foster B (eds) Doubled haploid production in crop plants. A Manual. FAO/IAEA Division, Wien, pp 367-390.

Forster BP, Thomas WTB (2005). Doubled haploids in genetics and plant breeding. Plant Breeding Rev 25: 57-88.

Grosse BA, Deimling S, Geiger HH (1996). Mapping of genes for anther culture ability in rye by molecular markers. Vortr Pflanzenzüchtg 35:282-283.

He P, Shen L, Lu C, Chen Y, Zhu L (1998). Analysis of quantitative trait loci which contribute to anther culturability in rice (Oryza sativa L). Mol Breeding 4: 165172.

Horvath H, Huang J, Wong OT, von Wettstein D (2002). Experiencies with genetic transformation of barley and characteristics of transgenic plants. In: Slafer GA, Molina-Cano JL, Savin R, Araus JL, Romagosa I (eds) Barley science: recent advances from molecular biology to agronomy of yield and quality. Food Products Press, New York, pp 143-174.

Hou L, Ullrich SE, Kleinhofs A (1994). Inheritance of anther culture traits in Barley. Crop Sci 34: 1243-1247.

Jacquard C, Asakaviciute R, Hamalian AM, Sangwan RS, Devaux P, Clément C (2006). Barley anther culture: effects of annual cycle and spike position on microspore embryogenesis and albinism. Plant Cell Rep 25: 375-381.

Komatsuda T, Annaka T, Oka S (1993). Genetic mapping of a quantitative trait locus (QTL) that enhances the shoot differentiation rate in Hordeum vulgare L. Theor Appl Genet 86: 713-720.

Kuenzel G, Korzum L, Meister A (2000). Cytologically integrated physical restriction fragment length polymorphism maps for the barley genome based on translocation breakpoints. Genetics 154: 397-412.

Kwon YS, Kim KM, Eun MY, Sohn JK (2002). QTL mapping and associated marker selection for the efficacy of green plant regeneration in anther culture of rice. Plant Breeding 121: 10-16.

Lander ES, Green P, Abrahamson J, Barlow A, Daly MJ, Lincoln SE, Newberg L (1987). Mapmaker: an interactive computer package for constructing primary genetic linkage maps of experimental and natural populations. Genomics 1: 174181. 
Larsen ET, Tuvesson IKD, Andersen SB (1991). Nuclear genes affecting percentage of green plants in barley (Hordeum vulgare L.) anther culture. Theor Appl Genet 82: 417-420.

Liu ZW, Biyashev RM, Saghai-Maroof MA (1996). Development of simple sequence repeat DNA markers and their integration into a barley linkage map. Theor Appl Genet 93: 869-876.

Magnard JL, Le Deunff E, Doménech J, Rogowsky PM, Testillano PS, Rougier M, Risueño MC, Vergne P, Dumas C (2000). Genes normally expressed in the endosperm are expressed at early stages of microspore embryogenesis in maize. Plant Mol Biol 44: 559-574.

Manninen OM (2000). Associations between anther-culture response and molecular markers on chromosome $2 \mathrm{H}, 3 \mathrm{H}$ and $4 \mathrm{H}$ of barley (Hordeum vulgare $\mathrm{L}$.). Theor Appl Genet 100: 57-62.

Mano Y, Takahashi H, Sato K, Takeda K (1996). Mapping genes for callus growth and shoot regeneration in barley (Hordeum vulgare L.). Breeding Sci 46: 137-142.

Maraschin SF, Caspers M, Potokina E, Wülfert F, Graner A, Spaink HP, Wang M (2006). cDNA array analysis of stress-induced gene expression in barley androgenesis. Physiol Plantarum 127: 535-550.

Muñoz-Amatriaín M, Svensson JT, Castillo AM, Cistué L, Close TJ, Vallés MP (2006). Transcriptome analysis of barley anthers: effect of mannitol treatment on microspore embryogenesis. Physiol Plantarum 127: 551-560.

Murigneux A, Bentolila S, Hardy T, Baud S, Guitton C, Jullien H, Ben Tahar S, Freyssinet G, Beckert M (1994). Genotypic variation of quantitative trait loci controlling in vitro androgenesis in maize. Genome 37: 970-976.

Powell W (1988). Diallel analysis of barley anther culture response. Genome 30: 152157.

Ramsay L, Macaulay M, degli Ivanissevich S, MacLean K, Cardle L, Fuller J, Edwards KJ, Tuvesson S, Morgante M, Massari A, Maestri E, Marmiroli N, Sjakste T, Ganal M, Powell W, Waugh R (2000). A simple sequence repeat-based linkage map of barley. Genetics 156: 1997-2005.

Reynolds TL, Crawford RL (1996). Changes in abundance of an abscisic acidresponsive, early cysteine-labeled metallothionein transcript during pollen embryogenesis in bread wheat (Triticum aestivum). Plant Mol Biol 32: 823-829. 
Sayed-Tabatabaei BE, Komatsuda T, Takaiwa F, Graner A (1999). DNA sequencing and primer designing for RFLP clones evenly distributed in the barley genome. Barley Genet Newsl 28: 15-18.

Szarejko I (2003). Doubled haploid mutation production. In: Maluszynski M, Kasha KJ, Forster BP, Szarejko I (eds) Doubled haploid production in crop plants, a manual. Kluwer Academic Publishers, Dordrecht, pp 351-362.

Tinker NA, Mather DE (1995). MQTL: software for simplified composite interval mapping of QTL in multiple environments. J Agric Genomics 1: (2).

Torp AM, Hansen AL, Anderson SB (2001). Chromosomal regions associated with green plant regeneration in wheat (Triticum aestivum L.) anther culture. Euphytica 119: 377-387.

Torp AM, Bekesiova I, Holme IB, Hansen AL, Andersen SB (2004). Genetics related to doubled haploid induction in vitro. In: Mujib A (ed) In vitro applications in crop improvement. Science Publishers, Playmouth, pp 34-52.

Vergne P, Riccardi F, Beckert M, Dumas C (1993). Identification of a 32-kDa anther marker protein for androgenic response in maize, Zea mays L. Theor Appl Genet 86: 843-850.

Vrinten PL, Nakamura T, Kasha KJ (1999). Characterization of cDNAs expressed in the early stages of microspore embryogenesis in barley (Hordeum vulgare) L. Plant Mol Biol 41: 455-463.

Yamagishi M, Otani M, Higashi M, Fukuta Y, Fukui K, Yano M, Shimada T (1998). Chromosomal regions controlling anther culturability in rice (Oryza sativa L). Euphytica 103: 227-234. 
Table 1. Mean values of the components of the anther culture response in parental cultivars Dobla and Igri, and in the DH population.

\begin{tabular}{lcccccc}
\hline \multirow{2}{*}{ Trait } & \multicolumn{2}{c}{ Parents } & & \multicolumn{3}{c}{ Doubled haploid population } \\
\cline { 2 - 3 } \cline { 5 - 7 } \cline { 5 - 7 } \cline { 5 - 7 } & Dobla & Igri & & $\mu$ & Minimum & Maximum \\
\hline nDM & 2121.0 & 2498.0 & & 1426.0 & 70.0 & 2714.0 \\
nEMB & 263.0 & $543.0^{* * a}$ & & 217.2 & 3.0 & 460.0 \\
nGP & 71.0 & $438.0^{* *}$ & & 88.0 & 0.0 & 370.0 \\
nAP & 70.0 & $13.0^{*}$ & & 73.3 & 1.0 & 264.0 \\
pGP & 49.6 & $97.1^{* *}$ & & 50.2 & 0.0 & 98.2 \\
\hline
\end{tabular}

${ }^{\text {a }}$ Significance of tests comparing the means of the parents: ${ }^{*} P<0.05,{ }^{*} P<0.01$ 
Table 2. Chromosome QTL location, the highest LOD value, allelic effect and percentage of the phenotypic variance explained for the individual locus $\left(R_{\mathrm{p}}^{2}\right)$ and multi-locus $\left(\mathrm{m} R_{\mathrm{p}}^{2}\right)$ in anther culture response.

\begin{tabular}{|c|c|c|c|c|c|c|}
\hline Trait & Chromosome & Marker interval & $\begin{array}{c}\text { LOD } \\
\text { scores }\end{array}$ & $\begin{array}{l}\text { Allelic } \\
\text { Effect }\end{array}$ & $\mathrm{R}_{\mathrm{p}}^{2}(\%)$ & $\underset{(\%)}{\mathrm{mR}^{2} \mathrm{p}}$ \\
\hline \multirow[t]{2}{*}{ nEMB } & $2 \mathrm{H}$ & vrs1-MWG822 & 4.8 & 152.2 & 40.92 & 52.47 \\
\hline & $6 \mathrm{H}$ & EBmac602 & 2.7 & 95.1 & 11.54 & \\
\hline \multirow[t]{2}{*}{ nGP } & $6 \mathrm{H}$ & EBmac602 & 3.7 & 96.5 & 32.68 & 50.53 \\
\hline & $5 \mathrm{H}$ & AS11-620 - Bmag812 & 2.8 & 77.1 & 17.85 & \\
\hline nAP & $2 \mathrm{H}$ & vrs1 & 2.8 & 67.1 & 24.94 & \\
\hline pGP & $5 \mathrm{H}$ & AS11-620 - Bmag812 & 3.2 & 31.0 & 21.07 & 21.07 \\
\hline
\end{tabular}


Fig. 1. Anther culture response QTLs mapped on the linkage map based on the cross between Dobla and Igri. Map distances, on the left side of the bars, are in centimorgans (cM). Distorted markers at $1 \%$ level are indicated by black boxes on the chromosome bars. Boxes on the left side of chromosomes indicate map intervals where QTLs were located.

$2 \mathrm{H}$

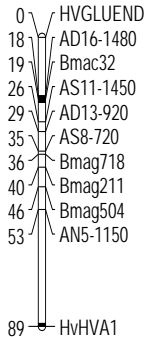

$3 \mathrm{H}$

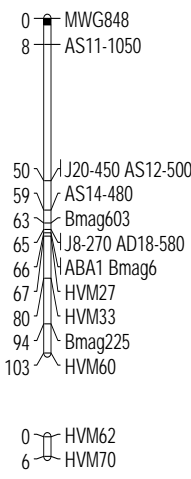

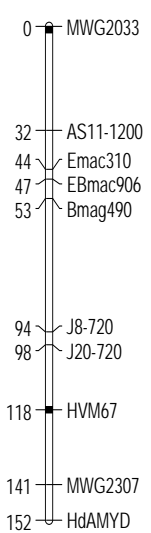

$5 \mathrm{H}$

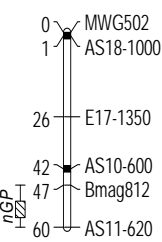

$0-1$ AD2-480 E17-420

21. $A D 2-820$

24 그 AD2-820

43- AF043094A
$6 \mathrm{H}$

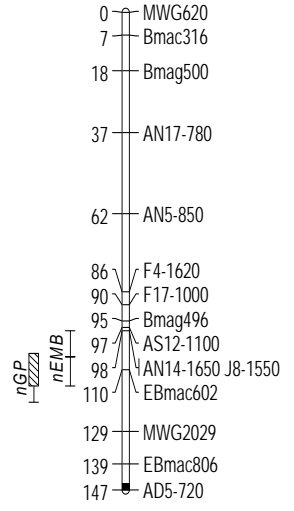

$147-$ AD5-720
29 EBmac603

0 - Bmac64

15-Bmag507

0) $\begin{aligned} & \mathrm{HVM} 11 \mathrm{~b} \\ & \mathrm{Bmac156}\end{aligned}$

15) HVGLB2

19. $\mid$ AS12-580 MWG2062

$$
\begin{aligned}
& \text { 0) CMWG658 } \\
& \begin{array}{r}
74 \text { Bmac40 } \\
14-1 \text { ABG711 AN13-1900 }
\end{array} \\
& \text { 23 AS16-900 } \\
& { }_{26} \mathcal{X}_{\text {AS3-580 }}
\end{aligned}
$$

